PROCEEDINGS OF THE

AMERICAN MATHEMATICAL SOCIETY

Volume 135, Number 6, June 2007, Pages 1669-1677

S 0002-9939(07)08712-6

Article electronically published on February 12, 2007

\title{
A STRUCTURE THEOREM FOR QUASI-HOPF COMODULE ALGEBRAS
}

\author{
FLORIN PANAITE AND FREDDY VAN OYSTAEYEN
}

(Communicated by Martin Lorenz)

\begin{abstract}
If $H$ is a quasi-Hopf algebra and $B$ is a right $H$-comodule algebra such that there exists $v: H \rightarrow B$ a morphism of right $H$-comodule algebras, we prove that there exists a left $H$-module algebra $A$ such that $B \simeq A \# H$. The main difference when comparing to the Hopf case is that, from the multiplication of $B$, which is associative, we have to obtain the multiplication of $A$, which in general is not; for this we use a canonical projection $E$ arising from the fact that $B$ becomes a quasi-Hopf $H$-bimodule.
\end{abstract}

\section{INTRODUCTION}

If $H$ is a Hopf algebra and $B$ is a right $H$-comodule algebra with the property that there exists a morphism $v: H \rightarrow B$ of right $H$-comodule algebras, then it is well known that $B$ is isomorphic as a right $H$-comodule algebra to a smash product $A \# H$, where $A$ is obtained as $A=B^{c o(H)}$ and its multiplication is the restriction of the multiplication of $B$.

On the other hand, if $H$ is a quasi-bialgebra and $A$ is a left $H$-module algebra, then the smash product $A \# H$ introduced in [1] becomes a right $H$-comodule algebra and the map $j: H \rightarrow A \# H, j(h)=1 \# h$, is a morphism of right $H$-comodule algebras. This raises the natural problem of checking whether for a quasi-Hopf algebra $H$ and a right $H$-comodule algebra $B$ such that there exists a morphism $v: H \rightarrow B$ of right $H$-comodule algebras, there exists a left $H$-module algebra $A$ such that $B \simeq A \# H$ as right $H$-comodule algebras. It is likely that $A$ appears as some sort of coinvariant of $B$, but it is clear that its multiplication cannot be obtained as the restriction of the one of $B$ (since $B$ is associative while in general $A$ is not), hence we need a different approach than in the Hopf case.

We first prove that $B$ becomes an object in the category ${ }_{H} \mathcal{M}_{H}^{H}$ of quasi-Hopf $H$ bimodules as introduced in [6]. An object $M$ in ${ }_{H} \mathcal{M}_{H}^{H}$ is endowed with a projection $E: M \rightarrow M$ and a concept of coinvariants, $M^{c o(H)}=E(M)$; applying this to $B$, we obtain a projection $E: B \rightarrow B$ and a subspace $B^{c o(H)}=E(B)$. Now we define the vector space $A=B^{c o(H)}$, with a multiplication defined by $a * a^{\prime}=E\left(a a^{\prime}\right)$, for

Received by the editors July 19, 2005 and, in revised form, March 7, 2006.

2000 Mathematics Subject Classification. Primary 16, 17.

This research was partially supported by the EC programme LIEGRITS, RTN 2003, 505078, and by the bilateral project "New techniques in Hopf algebras and graded ring theory" of the Flemish and Romanian Ministries of Research. The first author was also partially supported by the programme CERES of the Romanian Ministry of Education and Research, contract no. $4-147 / 2004$. 
all $a, a^{\prime} \in A$. The isomorphism $B \simeq A \# H$ follows from the structure theorem for quasi-Hopf $H$-bimodules, cf. [6], and we only have to prove that it is a morphism of right $H$-comodule algebras.

An application of our structure theorem is that, if we have a smash product $A \# H$ for a quasi-Hopf algebra $H$, it provides a method to get $A$ back from $A \# H$, as $A \simeq(A \# H)^{c o(H)}$.

\section{Preliminaries}

We work over a field $k$. All algebras, linear spaces, etc. will be over $k$; unadorned $\otimes$ means $\otimes_{k}$. Following Drinfeld [2], a quasi-bialgebra is a fourtuple $(H, \Delta, \varepsilon, \Phi)$, where $H$ is an associative algebra with unit, $\Phi$ is an invertible element in $H \otimes H \otimes H$, and $\Delta: H \rightarrow H \otimes H$ and $\varepsilon: H \rightarrow k$ are algebra homomorphisms satisfying the identities

$$
\begin{aligned}
& (i d \otimes \Delta)(\Delta(h))=\Phi(\Delta \otimes i d)(\Delta(h)) \Phi^{-1}, \\
& (i d \otimes \varepsilon)(\Delta(h))=h \otimes 1, \quad(\varepsilon \otimes i d)(\Delta(h))=1 \otimes h,
\end{aligned}
$$

for all $h \in H$, and $\Phi$ has to be a normalized 3-cocycle, in the sense that

$$
\begin{aligned}
& (1 \otimes \Phi)(i d \otimes \Delta \otimes i d)(\Phi)(\Phi \otimes 1)=(i d \otimes i d \otimes \Delta)(\Phi)(\Delta \otimes i d \otimes i d)(\Phi), \\
& (i d \otimes \varepsilon \otimes i d)(\Phi)=1 \otimes 1 \otimes 1 .
\end{aligned}
$$

The identities (1.2), (1.3) and (1.4) also imply that

$$
(\varepsilon \otimes i d \otimes i d)(\Phi)=(i d \otimes i d \otimes \varepsilon)(\Phi)=1 \otimes 1 \otimes 1 .
$$

The map $\Delta$ is called the coproduct or the comultiplication, $\varepsilon$ the counit and $\Phi$ the reassociator. We will use the version of Sweedler's sigma notation: $\Delta(h)=h_{1} \otimes h_{2}$, and since $\Delta$ is only quasi-coassociative we adopt the further convention

$$
(\Delta \otimes i d)(\Delta(h))=h_{(1,1)} \otimes h_{(1,2)} \otimes h_{2}, \quad(i d \otimes \Delta)(\Delta(h))=h_{1} \otimes h_{(2,1)} \otimes h_{(2,2)},
$$

for all $h \in H$. We will denote the tensor components of $\Phi$ by capital letters and those of $\Phi^{-1}$ by small letters, namely

$$
\begin{aligned}
& \Phi=X^{1} \otimes X^{2} \otimes X^{3}=T^{1} \otimes T^{2} \otimes T^{3}=Y^{1} \otimes Y^{2} \otimes Y^{3}=\cdots \\
& \Phi^{-1}=x^{1} \otimes x^{2} \otimes x^{3}=t^{1} \otimes t^{2} \otimes t^{3}=y^{1} \otimes y^{2} \otimes y^{3}=\cdots .
\end{aligned}
$$

The quasi-bialgebra $H$ is called a quasi-Hopf algebra if there exists an anti-automorphism $S$ of the algebra $H$ and elements $\alpha, \beta \in H$ such that, for all $h \in H$, we have:

$$
\begin{aligned}
& S\left(h_{1}\right) \alpha h_{2}=\varepsilon(h) \alpha \quad \text { and } \quad h_{1} \beta S\left(h_{2}\right)=\varepsilon(h) \beta, \\
& X^{1} \beta S\left(X^{2}\right) \alpha X^{3}=1 \quad \text { and } \quad S\left(x^{1}\right) \alpha x^{2} \beta S\left(x^{3}\right)=1 .
\end{aligned}
$$

The axioms for a quasi-Hopf algebra imply that $\varepsilon(\alpha) \varepsilon(\beta)=1$ so, by rescaling $\alpha$ and $\beta$, we may assume without loss of generality that $\varepsilon(\alpha)=\varepsilon(\beta)=1$ and $\varepsilon \circ S=\varepsilon$.

If $H$ is a quasi-Hopf algebra, following [4], 5] we may define the elements

(1.8) $p_{R}=p^{1} \otimes p^{2}=x^{1} \otimes x^{2} \beta S\left(x^{3}\right), \quad q_{R}=q^{1} \otimes q^{2}=X^{1} \otimes S^{-1}\left(\alpha X^{3}\right) X^{2}$, satisfying the relations (for all $h \in H$ ):

$$
\begin{gathered}
q_{1}^{1} p^{1} \otimes q_{2}^{1} p^{2} S\left(q^{2}\right)=1 \otimes 1, \quad q^{1} p_{1}^{1} \otimes S^{-1}\left(p^{2}\right) q^{2} p_{2}^{1}=1 \otimes 1, \\
\Delta\left(h_{1}\right) p_{R}\left[1 \otimes S\left(h_{2}\right)\right]=p_{R}[h \otimes 1], \quad\left[1 \otimes S^{-1}\left(h_{2}\right)\right] q_{R} \Delta\left(h_{1}\right)=[h \otimes 1] q_{R} .
\end{gathered}
$$


Let us record the following easy consequence of (1.7) (for $\left.q=q_{R}=q^{1} \otimes q^{2}\right)$ :

$$
q^{1} \beta S\left(q^{2}\right)=1
$$

Recall from [4] the notion of comodule algebra over a quasi-bialgebra.

Definition 1.1. Let $H$ be a quasi-bialgebra. A unital associative algebra $B$ is called a right $H$-comodule algebra if there exist an algebra morphism $\rho: B \rightarrow B \otimes H$ and an invertible element $\Phi_{\rho} \in B \otimes H \otimes H$ such that:

$$
\Phi_{\rho}(\rho \otimes i d)(\rho(b))=(i d \otimes \Delta)(\rho(b)) \Phi_{\rho}, \quad \forall b \in B,
$$

$$
\left(1_{B} \otimes \Phi\right)(i d \otimes \Delta \otimes i d)\left(\Phi_{\rho}\right)\left(\Phi_{\rho} \otimes 1_{H}\right)=(i d \otimes i d \otimes \Delta)\left(\Phi_{\rho}\right)(\rho \otimes i d \otimes i d)\left(\Phi_{\rho}\right),
$$

$$
(i d \otimes \varepsilon) \circ \rho=i d,
$$

$$
(i d \otimes \varepsilon \otimes i d)\left(\Phi_{\rho}\right)=(i d \otimes i d \otimes \varepsilon)\left(\Phi_{\rho}\right)=1_{B} \otimes 1_{H} .
$$

The first example of a right $H$-comodule algebra is $H$ itself, with $\rho=\Delta$ and $\Phi_{\rho}=\Phi$. For a right $H$-comodule algebra $\left(B, \rho, \Phi_{\rho}\right)$ we will denote $\rho(b)=b_{(0)} \otimes b_{(1)}$ for all $b \in B$. If $\left(B^{\prime}, \rho^{\prime}, \Phi_{\rho^{\prime}}\right)$ is another right $H$-comodule algebra, a morphism of right $H$-comodule algebras $f: B \rightarrow B^{\prime}$ is an algebra map such that $\rho^{\prime} \circ f=(f \otimes i d) \circ \rho$ and $\Phi_{\rho^{\prime}}=(f \otimes i d \otimes i d)\left(\Phi_{\rho}\right)$.

Suppose that $(H, \Delta, \varepsilon, \Phi)$ is a quasi-bialgebra. If $U, V, W$ are left $H$-modules, define $a_{U, V, W}:(U \otimes V) \otimes W \rightarrow U \otimes(V \otimes W)$ by

$$
a_{U, V, W}((u \otimes v) \otimes w)=\Phi \cdot(u \otimes(v \otimes w)) .
$$

The category ${ }_{H} \mathcal{M}$ of left $H$-modules becomes a monoidal category (see [7, 8 ] for the terminology) with tensor product $\otimes$ given via $\Delta$, associativity constraints $a_{U, V, W}$, unit $k$ as a trivial $H$-module and the usual left and right unit constraints.

Again let $H$ be a quasi-bialgebra. We say that a $k$-vector space $A$ is a left $H$ module algebra if it is an algebra in the monoidal category ${ }_{H} \mathcal{M}$, that is $A$ has a multiplication and a usual unit $1_{A}$ satisfying the following conditions:

$$
\begin{aligned}
& \left(a a^{\prime}\right) a^{\prime \prime}=\left(X^{1} \cdot a\right)\left[\left(X^{2} \cdot a^{\prime}\right)\left(X^{3} \cdot a^{\prime \prime}\right)\right], \\
& h \cdot\left(a a^{\prime}\right)=\left(h_{1} \cdot a\right)\left(h_{2} \cdot a^{\prime}\right), \\
& h \cdot 1_{A}=\varepsilon(h) 1_{A},
\end{aligned}
$$

for all $a, a^{\prime}, a^{\prime \prime} \in A$ and $h \in H$, where $h \otimes a \rightarrow h \cdot a$ is the left $H$-module structure of $A$. Following [1] we define the smash product $A \# H$ as follows: as vector space $A \# H$ is $A \otimes H$ (elements $a \otimes h$ will be written $a \# h$ ) with multiplication given by

$$
(a \# h)\left(a^{\prime} \# h^{\prime}\right)=\left(x^{1} \cdot a\right)\left(x^{2} h_{1} \cdot a^{\prime}\right) \# x^{3} h_{2} h^{\prime},
$$

for all $a, a^{\prime} \in A, h, h^{\prime} \in H$. Then $A \# H$ is an associative algebra with unit $1_{A} \# 1$. Moreover, by [1], $\left(A \# H, \rho, \Phi_{\rho}\right)$ becomes a right $H$-comodule algebra, with $\rho$ : $A \# H \rightarrow(A \# H) \otimes H, \rho(a \# h)=\left(x^{1} \cdot a \# x^{2} h_{1}\right) \otimes x^{3} h_{2}$ and $\Phi_{\rho}=\left(1 \# X^{1}\right) \otimes X^{2} \otimes X^{3}$. Also, it is easy to see that the map $j: H \rightarrow A \# H, j(h)=1 \# h$, is a morphism of right $H$-comodule algebras.

If $A, A^{\prime}$ are left $H$-module algebras, a map $f: A \rightarrow A^{\prime}$ is a morphism of left $H$-module algebras if it is multiplicative, unital and a morphism of left $H$-modules. 
If $H$ is a quasi-Hopf algebra, $B$ an associative algebra and $v: H \rightarrow B$ an algebra map, then, following [1], we can introduce on the vector space $B$ a left $H$-module algebra structure, denoted by $B^{v}$ in what follows, for which the multiplication, unit and left $H$-action are:

$$
\begin{aligned}
& b \circ b^{\prime}=v\left(X^{1}\right) b v\left(S\left(x^{1} X^{2}\right) \alpha x^{2} X_{1}^{3}\right) b^{\prime} v\left(S\left(x^{3} X_{2}^{3}\right)\right), \quad \forall b, b^{\prime} \in B, \\
& 1_{B^{v}}=v(\beta), \\
& h \triangleright_{v} b=v\left(h_{1}\right) b v\left(S\left(h_{2}\right)\right), \quad \forall h \in H, b \in B .
\end{aligned}
$$

If $H$ is a quasi-Hopf algebra and $A$ is a left $H$-module algebra, define the map

$$
i_{0}: A \rightarrow A \# H, \quad i_{0}(a)=p^{1} \cdot a \# p^{2}, \forall a \in A,
$$

where $p=p_{R}=p^{1} \otimes p^{2}$ is given by (1.8). Then, by [1], $i_{0}$ becomes a morphism of left $H$-module algebras from $A$ to $(A \# H)^{j}$.

\section{The STRUCTURE TheOREM}

We start with a lemma which is of independent interest.

Lemma 2.1. Let $H$ be a quasi-bialgebra and let $A$ be a left $H$-module with a multiplication. Define a multiplication on $A \otimes H$ by

$$
(a \otimes h)\left(a^{\prime} \otimes h^{\prime}\right)=\left(x^{1} \cdot a\right)\left(x^{2} h_{1} \cdot a^{\prime}\right) \otimes x^{3} h_{2} h^{\prime},
$$

for all $a, a^{\prime} \in A$ and $h, h^{\prime} \in H$, and assume that this multiplication is associative. Then:

(i) The multiplication of A satisfies the condition

$$
(a b) c=\left(X^{1} \cdot a\right)\left(\left(X^{2} \cdot b\right)\left(X^{3} \cdot c\right)\right), \quad \forall a, b, c \in A .
$$
then

(ii) If moreover $A$ has a usual unit $1_{A}$ satisfying $h \cdot 1_{A}=\varepsilon(h) 1_{A}$ for all $h \in H$,

$$
h \cdot(a b)=\left(h_{1} \cdot a\right)\left(h_{2} \cdot b\right),
$$

for all $h \in H$ and $a, b \in A$; that is, $A$ is a left $H$-module algebra, so the multiplication (2.1) is just the one of the smash product $A \# H$.

Proof. (i) Let $a, b, c \in A$; then one can easily compute that in $A \otimes H$ we have:

$$
\begin{aligned}
& ((a \otimes 1)(b \otimes 1))(c \otimes 1)=\left(y^{1} \cdot\left(\left(x^{1} \cdot a\right)\left(x^{2} \cdot b\right)\right)\right)\left(y^{2} x_{1}^{3} \cdot c\right) \otimes y^{3} x_{2}^{3}, \\
& (a \otimes 1)((b \otimes 1)(c \otimes 1))=\left(y^{1} \cdot a\right)\left(y^{2} \cdot\left(\left(x^{1} \cdot b\right)\left(x^{2} \cdot c\right)\right)\right) \otimes y^{3} x^{3} .
\end{aligned}
$$

Since $A \otimes H$ is associative, these are equal; by applying $\varepsilon$ on the second position, we obtain $(a b) c=\left(X^{1} \cdot a\right)\left(\left(X^{2} \cdot b\right)\left(X^{3} \cdot c\right)\right)$, q.e.d.

(ii) Let $a, b \in A$ and $h \in H$; write that $\left(\left(1_{A} \otimes h\right)(a \otimes 1)\right)(b \otimes 1)=\left(1_{A} \otimes h\right)((a \otimes 1)(b \otimes$ $1)$ ) in $A \otimes H$, then apply $\varepsilon$ in the second position and obtain $\left(h_{1} \cdot a\right)\left(h_{2} \cdot b\right)=h \cdot(a b)$, q.e.d.

The main ingredient for proving our structure theorem for quasi-Hopf comodule algebras will be the structure theorem for quasi-Hopf bimodules, so we recall first some facts from [6]. 
Let $H$ be a quasi-bialgebra and $M$ an $H$-bimodule together with an $H$-bimodule map $\rho: M \rightarrow M \otimes H$, with notation $\rho(m)=m_{(0)} \otimes m_{(1)}$ for $m \in M(\rho$ is called a right $H$-coaction on $M)$. Then $(M, \rho)$ is called a (right) quasi-Hopf $H$-bimodule if

$$
\begin{aligned}
& \left(i d_{M} \otimes \varepsilon\right) \circ \rho=i d_{M}, \\
& \Phi \cdot\left(\rho \otimes i d_{M}\right)(\rho(m))=\left(i d_{M} \otimes \Delta\right)(\rho(m)) \cdot \Phi, \quad \forall m \in M .
\end{aligned}
$$

The category of right quasi-Hopf $H$-bimodules will be denoted by ${ }_{H} \mathcal{M}_{H}^{H}$ (the morphisms in the category are the $H$-bimodule maps intertwining the $H$-coactions).

If $(V, \triangleright)$ is a left $H$-module, then $V \otimes H$ becomes a right quasi-Hopf $H$-bimodule with structure:

$$
\begin{aligned}
& a \cdot(v \otimes h) \cdot b=\left(a_{1} \triangleright v\right) \otimes a_{2} h b, \\
& \rho_{V \otimes H}(v \otimes h)=\left(x^{1} \triangleright v \otimes x^{2} h_{1}\right) \otimes x^{3} h_{2},
\end{aligned}
$$

for all $a, b, h \in H$ and $v \in V$.

Suppose now that $H$ is a quasi-Hopf algebra and $(M, \rho)$ is a right quasi-Hopf $H$-bimodule. Define the map $E: M \rightarrow M$ by

$$
E(m)=q^{1} \cdot m_{(0)} \cdot \beta S\left(q^{2} m_{(1)}\right), \quad \forall m \in M,
$$

where $q=q_{R}=q^{1} \otimes q^{2}$ is given by (1.8). Also, for $h \in H$ and $m \in M$, define

$$
h \triangleright m=E(h \cdot m) .
$$

Some properties of $E$ and $\triangleright$ are collected in [6], Proposition 3.4, for instance (for $h, h^{\prime} \in H$ and $\left.m \in M\right): E^{2}=E ; E(m \cdot h)=E(m) \varepsilon(h) ; h \triangleright E(m)=E(h \cdot m) \equiv h \triangleright m$; $\left(h h^{\prime}\right) \triangleright m=h \triangleright\left(h^{\prime} \triangleright m\right) ; h \cdot E(m)=\left(h_{1} \triangleright E(m)\right) \cdot h_{2} ; E\left(m_{(0)}\right) \cdot m_{(1)}=m$; $E\left(E(m)_{(0)}\right) \otimes E(m)_{(1)}=E(m) \otimes 1$.

Because of these properties, the following notions of coinvariants all coincide:

$$
M^{c o(H)}=E(M)=\{n \in M / E(n)=n\}=\left\{n \in M / E\left(n_{(0)}\right) \otimes n_{(1)}=E(n) \otimes 1\right\} .
$$

From the above properties it follows that $\left(M^{c o(H)}, \triangleright\right)$ is a left $H$-module.

Another description of $M^{c o(H)}$ is ([6], Corollary 3.9):

$$
M^{c o(H)}=\left\{n \in M / \rho(n)=\left(x^{1} \triangleright n\right) \cdot x^{2} \otimes x^{3}\right\} .
$$

For a quasi-Hopf $H$-bimodule of type $V \otimes H$, with $V \in{ }_{H} \mathcal{M}$, we have $(V \otimes H)^{c o(H)}=$ $V \otimes 1$ and $E(v \otimes h)=v \otimes \varepsilon(h) 1$, for all $v \in V$ and $h \in H$.

We can now state the structure theorem for quasi-Hopf $H$-bimodules.

Theorem 2.2 (6]). Let $H$ be a quasi-Hopf algebra and let $M$ be a right quasi-Hopf $H$-bimodule. Consider $V=M^{c o(H)}$ as a left $H$-module with $H$-action $\triangleright$ as in (2.7), and $V \otimes H$ as a right quasi-Hopf $H$-bimodule as above. Then the map

$$
\nu: V \otimes H \rightarrow M, \quad \nu(v \otimes h)=v \cdot h, \quad \forall v \in V \text { and } h \in H,
$$

provides an isomorphism of right quasi-Hopf $H$-bimodules, with inverse

$$
\nu^{-1}: M \rightarrow V \otimes H, \quad \nu^{-1}(m)=E\left(m_{(0)}\right) \otimes m_{(1)}, \forall m \in M .
$$

From now on we fix a quasi-Hopf algebra $H$ and a right $H$-comodule algebra $\left(B, \rho, \Phi_{\rho}\right)$, with notation $\rho(b)=b_{(0)} \otimes b_{(1)} \in B \otimes H$, such that there exists $v$ : $H \rightarrow B$, a morphism of right $H$-comodule algebras (in particular, this implies $\rho(v(h))=v\left(h_{1}\right) \otimes h_{2}$, for all $h \in H$, and $\left.\Phi_{\rho}=v\left(X^{1}\right) \otimes X^{2} \otimes X^{3}\right)$.

Lemma 2.3. $(B, \rho)$ becomes an object in ${ }_{H} \mathcal{M}_{H}^{H}$. 
Proof. First, $B$ becomes an $H$-bimodule via $v$ (i.e. $h \cdot b \cdot h^{\prime}=v(h) b v\left(h^{\prime}\right)$ for all $h, h^{\prime} \in H$ and $\left.b \in B\right)$. We prove now that $\rho: B \rightarrow B \otimes H$ is an $H$-bimodule map. We compute:

$$
\begin{aligned}
\rho\left(h \cdot b \cdot h^{\prime}\right) & =\rho\left(v(h) b v\left(h^{\prime}\right)\right) \\
& =\rho(v(h)) \rho(b) \rho\left(v\left(h^{\prime}\right)\right) \\
& =\left(v\left(h_{1}\right) \otimes h_{2}\right)\left(b_{(0)} \otimes b_{(1)}\right)\left(v\left(h_{1}^{\prime}\right) \otimes h_{2}^{\prime}\right) \\
& =v\left(h_{1}\right) b_{(0)} v\left(h_{1}^{\prime}\right) \otimes h_{2} b_{(1)} h_{2}^{\prime} \\
& =h_{1} \cdot b_{(0)} \cdot h_{1}^{\prime} \otimes h_{2} b_{(1)} h_{2}^{\prime} \\
& =h \cdot \rho(b) \cdot h^{\prime}, \quad \text { q.e.d. }
\end{aligned}
$$

Obviously we have $\left(i d_{B} \otimes \varepsilon\right) \circ \rho=i d_{B}$. Finally, it is easy to see that

$$
\Phi \cdot\left(\rho \otimes i d_{B}\right)(\rho(b))=\left(i d_{B} \otimes \Delta\right)(\rho(b)) \cdot \Phi
$$

because this is exactly the condition

$$
\Phi_{\rho}\left(\rho \otimes i d_{B}\right)(\rho(b))=\left(i d_{B} \otimes \Delta\right)(\rho(b)) \Phi_{\rho}
$$

from the definition of a right $H$-comodule algebra, due to the fact that $\Phi_{\rho}=$ $v\left(X^{1}\right) \otimes X^{2} \otimes X^{3}$. Hence $(B, \rho)$ is indeed a right quasi-Hopf $H$-bimodule.

Since $B$ is an object in ${ }_{H} \mathcal{M}_{H}^{H}$, we can consider the map $E: B \rightarrow B$, which is given by

$$
E(b)=v\left(q^{1}\right) b_{(0)} v\left(\beta S\left(q^{2} b_{(1)}\right)\right), \quad \forall b \in B,
$$

where $q=q_{R}=q^{1} \otimes q^{2}$ is given by (1.8), and we can take the coinvariants

$$
B^{c o(H)}=E(B)=\{b \in B / E(b)=b\}=\left\{b \in B / E\left(b_{(0)}\right) \otimes b_{(1)}=E(b) \otimes 1\right\} .
$$

The $H$-module algebra $A$ we are looking for will be, as a vector space, $A=B^{\operatorname{co}(H)}$.

We have the $H$-action on $B$ given by $h \triangleright b=E(v(h) b)$, which gives a left $H$ module structure on $A$. Let us note that, because of (1.11), we have $E(1)=1$, hence $1 \in A$. By the structure theorem for quasi-Hopf $H$-bimodules, we know that the map

$$
\Psi: A \otimes H \rightarrow B, \quad \Psi(a \otimes h)=a v(h),
$$

is an isomorphism in ${ }_{H} \mathcal{M}_{H}^{H}$ (the left $H$-module structure of $A$ is $\triangleright$ ), with inverse

$$
\Psi^{-1}: B \rightarrow A \otimes H, \quad \Psi^{-1}(b)=E\left(b_{(0)}\right) \otimes b_{(1)} .
$$

Our aim will be to introduce a new multiplication on $A$, denoted by $*$, such that $(A, *, 1, \triangleright)$ becomes a left $H$-module algebra and $\Psi$ becomes an isomorphism of right $H$-comodule algebras between $A \# H$ and $B$ (note that $\Psi$ has the property that $\Psi \circ j=v$, where $j$ is the canonical map $H \rightarrow A \# H)$. We will define actually a new multiplication $*$ on the whole $B$, and will take its restriction to $A$. Namely, for all $b, b^{\prime} \in B$, define

$$
b * b^{\prime}=E\left(b b^{\prime}\right) .
$$

Since $A=E(B), *$ restricts to a multiplication on $A$. Since for $a \in A$ we have $E(a)=a$, we obtain $a * 1=1 * a=E(a)=a$, hence 1 is a unit for $(A, *)$. Now let 
$h \in H$; we compute:

$$
\begin{aligned}
h \triangleright 1 & =E(v(h)) \\
& =v\left(q^{1}\right) v(h)_{(0)} v\left(\beta S\left(q^{2} v(h)_{(1)}\right)\right) \\
& =v\left(q^{1}\right) v\left(h_{1}\right) v\left(\beta S\left(q^{2} h_{2}\right)\right) \\
& =v\left(q^{1} h_{1} \beta S\left(h_{2}\right) S\left(q^{2}\right)\right) \\
\text { (1.6) } & =v\left(q^{1} \beta S\left(q^{2}\right)\right) \varepsilon(h) \\
& =\varepsilon(h) 1 .
\end{aligned}
$$

In view of Lemma 2.1 in order to get that $(A, *, 1, \triangleright)$ is a left $H$-module algebra, it is enough to prove that the multiplication defined on $A \otimes H$ by

$$
(a \otimes h)\left(a^{\prime} \otimes h^{\prime}\right)=\left(x^{1} \triangleright a\right) *\left(x^{2} h_{1} \triangleright a^{\prime}\right) \otimes x^{3} h_{2} h^{\prime}
$$

is associative. Since $\Psi: A \otimes H \rightarrow B$ is bijective and $B$ is associative, it is enough to prove that $\Psi$ is multiplicative, that is, for all $a, a^{\prime} \in A$ and $h, h^{\prime} \in H$ :

$$
\Psi\left(\left(x^{1} \triangleright a\right) *\left(x^{2} h_{1} \triangleright a^{\prime}\right) \otimes x^{3} h_{2} h^{\prime}\right)=\Psi(a \otimes h) \Psi\left(a^{\prime} \otimes h^{\prime}\right) .
$$

We first prove a relation that will be used in the proof of the multiplicativity of $\Psi$.

Lemma 2.4. Let $H$ be a quasi-Hopf algebra; then we have:

$$
q_{1}^{1} t^{1} x^{1} \otimes q_{(2,1)}^{1} t_{1}^{2} z^{1} x^{2} \otimes q_{(2,2)}^{1} t_{2}^{2} z^{2} \beta S\left(q^{2} t^{3} z^{3}\right) x^{3}=1 \otimes 1 \otimes 1,
$$

where $q=q_{R}=q^{1} \otimes q^{2}$ is given by (1.8).

Proof. We will also use the element $p=p_{R}=p^{1} \otimes p^{2}$ given by (1.8). We compute:

$$
\begin{aligned}
q_{1}^{1} t^{1} x^{1} \otimes q_{(2,1)}^{1} & t_{1}^{2} z^{1} x^{2} \otimes q_{(2,2)}^{1} t_{2}^{2} z^{2} \beta S\left(q^{2} t^{3} z^{3}\right) x^{3} \\
& =q_{1}^{1} Z^{1} t_{1}^{1} y^{1} x^{1} \otimes q_{(2,1)}^{1} Z^{2} t_{2}^{1} y^{2} x^{2} \otimes q_{(2,2)}^{1} Z^{3} t^{2} y_{1}^{3} \beta S\left(q^{2} t^{3} y_{2}^{3}\right) x^{3} \\
& =q_{1}^{1} Z^{1} t_{1}^{1} x^{1} \otimes q_{(2,1)}^{1} Z^{2} t_{2}^{1} x^{2} \otimes q_{(2,2)}^{1} Z^{3} t^{2} \beta S\left(q^{2} t^{3}\right) x^{3} \\
\text { (1.5) } & =Z^{1} q_{(1,1)}^{1} p_{1}^{1} x^{1} \otimes Z^{2} q_{(1,2)}^{1} p_{2}^{1} x^{2} \otimes Z^{3} q_{2}^{1} p^{2} S\left(q^{2}\right) x^{3} \\
& =Z^{1} x^{1} \otimes Z^{2} x^{2} \otimes Z^{3} x^{3} \\
& =1 \otimes 1 \otimes 1,
\end{aligned}
$$

and the relation is proved.

We will need two of the general properties of the map $E$ on a right quasi-Hopf $H$-bimodule $M$ recalled before, which for $M=B$ become:

$$
v(h) a=\left(h_{1} \triangleright a\right) v\left(h_{2}\right), \quad \forall h \in H, a \in A,
$$

and, if $a \in B$, then

$$
a \in A \Longleftrightarrow a_{(0)} \otimes a_{(1)}=\left(x^{1} \triangleright a\right) v\left(x^{2}\right) \otimes x^{3} .
$$

We also need a more explicit formula for $a * a^{\prime}$, if $a, a^{\prime} \in A$. We can write:

$$
\begin{aligned}
a * a^{\prime} & =E\left(a a^{\prime}\right) \\
& =v\left(q^{1}\right) a_{(0)} a_{(0)}^{\prime} v\left(\beta S\left(q^{2} a_{(1)} a_{(1)}^{\prime}\right)\right) \\
(2.17) & =v\left(q^{1}\right)\left(t^{1} \triangleright a\right) v\left(t^{2}\right)\left(z^{1} \triangleright a^{\prime}\right) v\left(z^{2} \beta S\left(q^{2} t^{3} z^{3}\right)\right) .
\end{aligned}
$$


We can finally prove that $\Psi$ is multiplicative. We compute (for $a, a^{\prime} \in A$ and $\left.h, h^{\prime} \in H\right)$ :

$$
\begin{gathered}
\Psi(a \otimes h) \Psi\left(a^{\prime} \otimes h^{\prime}\right)=a v(h) a^{\prime} v\left(h^{\prime}\right) \\
(\underline{2.16})=a\left(h_{1} \triangleright a^{\prime}\right) v\left(h_{2} h^{\prime}\right), \\
\Psi\left(\left(x^{1} \triangleright a\right) *\left(x^{2} h_{1} \triangleright a^{\prime}\right) \otimes x^{3} h_{2} h^{\prime}\right) \\
=\left(x^{1} \triangleright a\right) *\left(x^{2} h_{1} \triangleright a^{\prime}\right) v\left(x^{3} h_{2} h^{\prime}\right) \\
=v\left(q^{1}\right)\left(t^{1} x^{1} \triangleright a\right) v\left(t^{2}\right)\left(z^{1} x^{2} h_{1} \triangleright a^{\prime}\right) v\left(z^{2} \beta S\left(q^{2} t^{3} z^{3}\right) x^{3} h_{2} h^{\prime}\right) \\
=\left(q_{1}^{1} t^{1} x^{1} \triangleright a\right) v\left(q_{2}^{1} t^{2}\right)\left(z^{1} x^{2} h_{1} \triangleright a^{\prime}\right) v\left(z^{2} \beta S\left(q^{2} t^{3} z^{3}\right) x^{3} h_{2} h^{\prime}\right) \\
\left(\underline{2.16)}=\left(q_{1}^{1} t^{1} x^{1} \triangleright a\right)\left(q_{(2,1)}^{1} t_{1}^{2} z^{1} x^{2} h_{1} \triangleright a^{\prime}\right) v\left(q_{(2,2)}^{1} t_{2}^{2} z^{2} \beta S\left(q^{2} t^{3} z^{3}\right) x^{3} h_{2} h^{\prime}\right)\right. \\
=a\left(h_{1} \triangleright a^{\prime}\right) v\left(h_{2} h^{\prime}\right), \quad \text { q.e.d. }
\end{gathered}
$$

Since obviously we have $\Psi(1 \otimes 1)=1$, now we have that $(A, *, 1, \triangleright)$ is a left $H$-module algebra and $\Psi: A \# H \rightarrow B$ is an algebra isomorphism. Using (2.17), the fact that $\rho(v(h))=v\left(h_{1}\right) \otimes h_{2}$ and the formula $\rho_{A \# H}(a \# h)=\left(x^{1} \triangleright a \# x^{2} h_{1}\right) \otimes x^{3} h_{2}$, one can easily see that $\rho_{B} \circ \Psi=(\Psi \otimes i d) \circ \rho_{A \# H}$. Moreover, since $\Phi_{A \# H}=\left(1 \# X^{1}\right) \otimes X^{2} \otimes X^{3}$ and $\Psi\left(1 \# X^{1}\right) \otimes X^{2} \otimes X^{3}=v\left(X^{1}\right) \otimes X^{2} \otimes X^{3}=\Phi_{B}$, we conclude that $\Psi$ is an isomorphism of right $H$-comodule algebras. Hence, we have proved the desired structure theorem:

Theorem 2.5. Let $H$ be a quasi-Hopf algebra and let $B$ be a right $H$-comodule algebra such that there exists $v: H \rightarrow B$, a morphism of right $H$-comodule algebras. Then there exists a left $H$-module algebra $A$ (whose structure is described above) such that $B \simeq A \# H$ as right $H$-comodule algebras.

Now let $H, B$ and $v: H \rightarrow B$ be as above. Since $B$ is an associative algebra and $v$ is an algebra map, we can consider the left $H$-module algebra $B^{v}$ as in the Preliminaries.

Proposition 2.6. With notation as above, the map

$$
\theta: A \rightarrow B^{v}, \quad \theta(a)=\left(p^{1} \triangleright a\right) v\left(p^{2}\right),
$$

where $p=p_{R}=p^{1} \otimes p^{2}$ is given by (1.8), is an injective morphism of left H-module algebras.

Proof. Since $\Psi: A \# H \rightarrow B$ is an algebra map satisfying $\Psi \circ j=v$, by [10], Lemma 4.1. it follows that $\Psi:(A \# H)^{j} \rightarrow B^{v}$ is a morphism of left $H$-module algebras. We know from the Preliminaries that the map $i_{0}: A \rightarrow(A \# H)^{j}, i_{0}(a)=p^{1} \triangleright a \otimes p^{2}$ is also a morphism of left $H$-module algebras, and one can see that actually $\theta=\Psi \circ i_{0}$, hence $\theta$ is indeed a morphism of left $H$-module algebras, and it is injective since $i_{0}$ is injective and $\Psi$ is bijective.

Note that in the Hopf case $\theta$ is simply the inclusion of $A$ into $B^{v}$.

Remark 2.7. Let $H$ be a quasi-Hopf algebra, let $A$ be a left $H$-module algebra and let $B=A \# H$; then, for this $B$ together with the canonical map $j: H \rightarrow A \# H$, one can show that the map $E$ is given by $E(a \# h)=\varepsilon(h)(a \# 1)$, and so $B^{c o(H)}=A \# 1$, with multiplication and $H$-action:

$$
\begin{aligned}
& (a \# 1) *\left(a^{\prime} \# 1\right)=a a^{\prime} \# 1, \quad \forall a, a^{\prime} \in A, \\
& h \triangleright(a \# 1)=h \cdot a \# 1, \quad \forall h \in H \quad \text { and } a \in A,
\end{aligned}
$$


that is $B^{c o(H)} \simeq A$ as left $H$-module algebras. Hence, the structure theorem allows to recover the structure of $A$ from the one of $A \# H$.

Remark 2.8. Let $H, B, v$ be as in Theorem 2.5. An obvious consequence of the theorem is that the category ${ }_{B} \mathcal{M}$ of left $B$-modules is equivalent to the category ${ }_{A, H} \mathcal{M}$ of left $A$-modules inside the monoidal category ${ }_{H} \mathcal{M}$. As suggested by the referee, this consequence may be rephrased as follows: for the category ${ }_{B} \mathcal{M}$, considered as a module category over the monoidal category ${ }_{H} \mathcal{M}$ (in the sense of 9 ), there exists an algebra $A$ in ${ }_{H} \mathcal{M}$ such that ${ }_{B} \mathcal{M}$ is equivalent to the category of left $A$-modules inside ${ }_{H} \mathcal{M}$; this last statement is a particular case of a general phenomenon concerning module categories over monoidal categories, see [3].

\section{REFERENCES}

[1] D. Bulacu, F. Panaite and F. Van Oystaeyen, Quasi-Hopf algebra actions and smash products, Comm. Algebra 28 (2000), 631-651. MR.1736752 (2000m:16048)

[2] V. G. Drinfeld, Quasi-Hopf algebras, Leningrad Math. J. 1 (1990), 1419-1457. MR1047964 (91b:17016)

[3] P. Etingof and V. Ostrik, Finite tensor categories, Mosc. Math. J. 4 (2004), 627-654, 782-783. MR2119143 (2005j:18006)

[4] F. Hausser and F. Nill, Diagonal crossed products by duals of quasi-quantum groups, Rev. Math. Phys. 11 (1999), 553-629. MR1696105 (2000d:81069)

[5] F. Hausser and F. Nill, Doubles of quasi-quantum groups, Comm. Math. Phys. 199 (1999), 547-589. MR1669685 (2000a:16075)

[6] F. Hausser and F. Nill, Integral theory for quasi-Hopf algebras, arXiv:math.QA/9904164.

[7] C. Kassel, "Quantum groups", Graduate Texts in Mathematics 155, Springer-Verlag, Berlin, 1995. MR1321145 (96e:17041)

[8] S. Majid, "Foundations of quantum group theory", Cambridge Univ. Press, 1995. MR1381692 (97g:17016)

[9] V. Ostrik, Module categories, weak Hopf algebras and modular invariants, Transform. Groups 8 (2003), 177-206. MR 1976459 (2004h:18006)

[10] F. Panaite and F. Van Oystaeyen, Quasi-Hopf algebras and representations of octonions and other quasialgebras, J. Math. Phys. 45 (2004), 3912-3929. MR2095679 (2005i:16079)

Institute of Mathematics of the Romanian Academy, PO-Box 1-764, RO-014700 Bucharest, Romania

E-mail address: Florin.Panaite@imar.ro

Department of Mathematics and Computer Science, University of Antwerp, MiddelHeimlaAn 1, B-2020 ANTwERP, Belgium

E-mail address: Francine.Schoeters@ua.ac.be 